\title{
BIÓPSIA HEPÁTICA NO PÓS-TRANSPLANTE DE FÍGADO: RELATO DE COMPLICAÇÃO E REVISÃO DA LITERATURA
}

\author{
Liver biopsy after liver transplantation: reported complication and literature review \\ Julia Girardi Cutovoi'; José Antonio Possatto Ferrer ${ }^{\text {; }}$ Elaine Cristina Ataide ${ }^{1}$; Jazon Romilson de Souza Almeida²; \\ Tiago Sevá Pereira²; Ilka Fátima Santana Ferreira Boin¹.
}

\section{RESUMO}

A biópsia hepática percutânea é um procedimento rotineiramente utilizado no pós-operatório de transplante hepático na investigação de causas de aumento enzimático; apresenta taxa total de complicações de 0,9\% a 3,7 \%, sendo a principal delas hemorragia. Relatamos o caso de um paciente do sexo masculino de 52 anos com diagnóstico de cirrose hepática por hepatite $\mathrm{C}$ e álcool submetido a transplante hepático com enxerto inteiro pela técnica de piggy back há 20 meses. Evoluiu sem complicações no pós-operatório e iniciou seguimento ambulatorial no Hospital de Clínicas da Unicamp (HC-Unicamp). Como apresentava difícil adequação aos imunossupressores, optou-se por submetê-lo à biópsia hepática percutânea em regime hospitalar para avaliação de possível rejeição celular. Evoluiu um dia após a biópsia com dor abdominal e aumento significativo de enzimas hepáticas, além de queda de três pontos de hemoglobina, tendo sido diagnosticado grande hematoma hepático subcapsular por Tomografia Computadorizada Multislice de abdome. Houve boa evolução com tratamento não operatório do hematoma, necessitando de transfusão de apenas um concentrado de hemácias, sem necessidade de transfusão de outros hemoterápicos. Permaneceu em observação hospitalar por sete dias, tendo tido alta em boas condições e reiniciando seguimento ambulatorial. A biópsia percutânea de fígado é procedimento invasivo, porém muito importante na avaliação e seguimento de pacientes transplantados hepáticos, podendo influenciar na terapia imunossupressora, assim como diagnosticar quadros de recidiva viral e possibilitar tratamento nesses casos. No entanto, não é isenta de complicações inerentes ao procedimento, como aqui relatado, devendo sua indicação ser sempre bem avaliada e neste caso pode ser conduzida de forma conservadora.

Keywords: Biópsia por Agulha Fina; Transplante Hepático; Hematoma; Complicações.

\footnotetext{
Instituições:

${ }^{1}$ Departamento de Cirurgia da Faculdade de Ciências Médicas da Universidade Estadual de Campinas (UNICAMP), Campinas/SP

2 Departamento de Gastroenterologia da Faculdade de Ciências Médicas da Universidade Estadual de Campinas (UNICAMP), Campinas/SP
}

\section{Correspondência:}

Julia Girardi Cutovoi

Rua Pedro Vieira da Silva, 64/43, CEP 13080-570, Campinas/SP

Tel.: (19) 98138-4444

E-mail: juliacutovoi@gmail.com
Aceito em: 05/12/2013

\section{INTRODUÇÃO}

A biópsia percutânea hepática é considerada padrão ouro para avaliação de desordens hepáticas agudas e crônicas. É rotineiramente realizada em pacientes transplantados, na avaliação de aumentos enzimáticos de etiologia indeterminada. Pode oferecer diagnóstico do grau de progressão e resposta ao tratamento de moléstias hepáticas. No caso de pacientes transplantados, avalia a resposta à terapia imunossupressora e possível recidiva viral. ${ }^{1-5}$

Geralmente, é procedimento seguro, conferindo risco mínimo de complicações, mesmo em pacientes hepatopatas crônicos, na avaliação de lesões focais e após transplante hepático, complicações essas que requerem hospitalização e que ocorrem em $0,13 \%$ a $5,4 \%$ das biópsia percutâneas. ${ }^{2,5}$ 
Especificamente em relação ao pós-operatório de transplante hepático, há poucos relatos na literatura descrevendo as complicações após biópsias percutâneas. Não há consenso em relação à melhor conduta frente a acidentes de punção decorrentes de biópsia percutânea em pacientes transplantados hepáticos, bem como não há descrição estatística quanto aos riscos desse procedimento nesses pacientes na literatura.

Relatamos o caso de paciente submetido à biópsia percutânea hepática após transplante hepático em acompanhamento na Unidade de Transplante Hepático do Hospital de Clínicas da Faculdade de Ciências Médicas/ Universidade Estadual de Campinas (FCM-Unicamp).

\section{Relato de Caso}

Paciente de 52 anos com diagnóstico de cirrose hepática por hepatite $\mathrm{C}$ e álcool, foi submetido a transplante hepático total em 14/10/2011, sob técnica de Piggy Back. O paciente evoluiu sem complicações no pós-operatório e recebeu alta da UTI no quinto dia pós-operatório e alta hospitalar no sétimo dia pós-operatório, mantendo seguimento ambulatorial.

Durante o seguimento, 45 dias após o transplante, apresentou, níveis aumentados das enzimas canaliculares, com GGT (Gama Glutamiltransferase) de $1048 \mathrm{U} / \mathrm{L}$ e FALC (Fosfatase Alcalina) de $177 \mathrm{U} / \mathrm{L}$, tendo sido então submetido à ultrassonografia (US) de abdome, que não demonstrou alterações morfológicas vasculares. Dois meses após o transplante, foi submetido à ressonância magnética de abdome, que demonstrou fígado de dimensões normais, parênquima com sinal homogêneo, anastomose portal e caval pérveas, veias hepáticas pérveas, sem dilatação de vias biliares. Apesar do manejo das medicações imunossupressoras, os níveis enzimáticos mantiveram-se aumentados até seis meses após o transplante (Aspartato Aminotrasnferase (AST) $359 \mathrm{U} / \mathrm{L}$, Alanina Aminotransferase (ALT) $732 \mathrm{U} / \mathrm{L}$, FALC 144 U/L, GGT 997 U/L), tendo-se optado então por realizar pulso com metilprednisolona, mantido com micofenolato sódico como imunossupressor, tendo sido introduzido everolimus em seu seguimento ambulatorial. Apresentou melhora dos níveis enzimáticos (AST 180 U/L, ALT 120 U/L, GGT 130 U/L, FALC 125 U/L).

Dezoito meses após o transplante, expressou novo aumento de enzimas hepáticas (AST 302 U/L, ALT 251 U/L, GGT 349 U/L, FALC 197 U/L), nesse momento, em uso de everolimus e tacrolimus como imunossupressores. Optou-se por submetê-lo à biópsia hepática para avaliar hipótese de rejeição do enxerto. Foi internado e submetido à biópsia percutânea hepática guiada por ultrassonografia. Foi utilizada agulha de biópsia VELOX de 14G, do tipo Tru-cut (MEDAX, Itália). Avaliação prévia da biópsia não mostrou discrasias sanguíneas, com hemoglobina $(\mathrm{Hb})$ de $11,3 \mathrm{mg} / \mathrm{dl}$, plaquetas (plaq) de 220000 e tempo de ativação de protrombina, demonstrado pela razão normalizada internacional (RNI) de 1,02. Após realização da biópsia evoluiu com dor abdominal em hipocôndrio direito e aumento importante das enzimas hepáticas: ALT 1266 U/L, AST 2290 U/L, com queda de três pontos da hemoglobina sérica $(\mathrm{Hb}$ de $8,1 \mathrm{mg} / \mathrm{dl}$ ). Submetido à ultrassonografia, verificou-se extensa área heterogênea sem fluxo ao Doppler centrada nos segmentos anteriores do lobo hepático direito, segmentos V e VIII, sem trombose arterial ou portal. Foi realizada então tomografia computadorizada multislice (TC) de abdome, que confirmou extenso hematoma (913 $\mathrm{ml}$ ) nesses segmentos, com necrose peri-hematoma intra-hepática nos segmentos adjacentes hepáticos, sem rompimento da cápsula hepática (Figuras 1 e 2).

Figure 1 - Tomografia computadorizada mostrando hematoma após biópsia guiada por US.

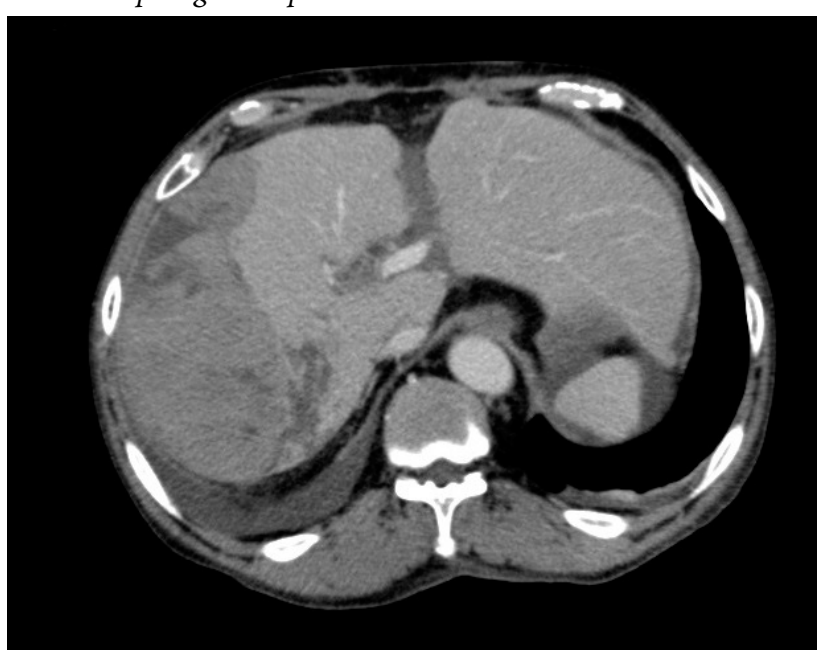

Figure 2 - Tomografia Computadorizada mostrando hematoma após biópsia guiada por US (corte coronal).

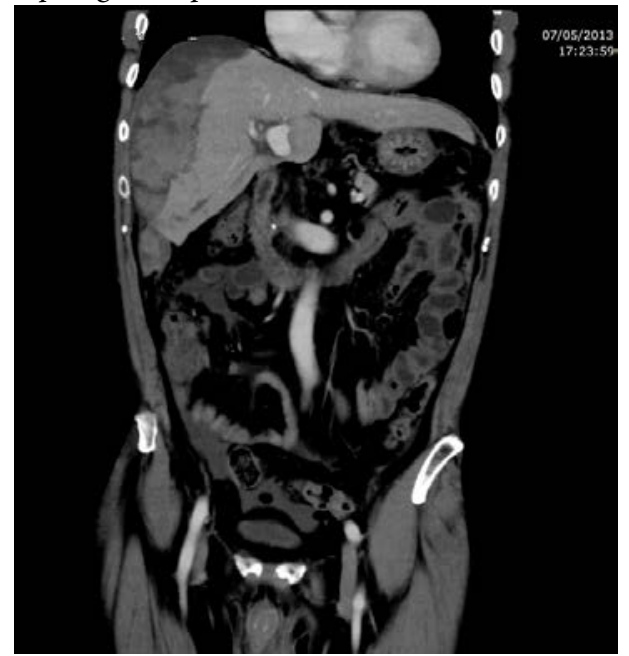


Optou-se por conduta conservadora, com tratamento não operatório do hematoma hepático. O paciente evoluiu com melhora dos níveis de hemoglobina com repouso absoluto e transfusão de um concentrado de hemácias. A TC de abdome de controle sete dias após mostrou estabilidade do hematoma. Recebeu alta no oitavo dia após a biópsia com melhora dos níveis de enzimas hepáticas (ALT 224 U/L, AST 113 U/L, GGT 217 U/L e FALC $179 \mathrm{U} / \mathrm{L})$, assintomático.

Realizada tomografia de controle trinta dias após a biópsia, foi verificada reabsorção parcial do hematoma, com $342 \mathrm{ml}$, mantendo níveis enzimáticos semelhantes aos níveis prévios à biópsia hepática.

A biópsia hepática demonstrou hepatite crônica pelo vírus C (recidiva viral no enxerto), com moderada atividade de interface e ausência de alterações histológicas sugestivas de rejeição.

O paciente segue com acompanhamento ambulatorial, com exames de imagem de controle mostrando reabsorção do hematoma hepático. Será avaliado para tratamento farmacológico de recidiva viral pela equipe de Moléstias Infecciosas do grupo de Transplante Hepático do HC-Unicamp.

\section{DISCUSSÃO}

A biópsia hepática percutânea foi realizada pela primeira vez na Alemanha, em 1883, por Paul Ehrlich. ${ }^{6}$ Devido à alta taxa de complicações, o procedimento só foi adotado globalmente a partir de 1957, com a técnica descrita por Menghini, ${ }^{7}$ a qual é realizada por punção, com tempo reduzido de permanência da agulha no interior do parênquima hepático (menos de um segundo). Desde então, biópsias hepáticas percutâneas são importantes ferramentas na prática diagnóstica e terapêutica.

A biópsia hepática percutânea guiada porultrassonografia (US) é considerada procedimento relativamente seguro, com taxas mais altas de complicação, de $0,13 \%$ a 5,4\%, sendo a principal complicação a hemorragia. $\mathrm{O}$ risco de sangramento varia de $0,06 \%$ a $1,7 \%$, e o risco de morte, geralmente causada por sangramento, de $0,0 \%$ a $0,48 \% .^{2,8}$ Biópsias realizadas em pacientes previamente submetidos a transplante hepático apresentam taxas de complicações similares. ${ }^{9,10}$ O sucesso e a reduzida taxa de complicações dependem da técnica utilizada na punção, do tipo e calibre da agulha utilizada, da experiência do profissional e da condição clínica do paciente. Há risco aumentado para sangramento associado à idade avançada, cirrose hepática ou presença de discrasias sanguíneas. Sangramento significativo pode ocorrer na forma de hematoma hepático, intraparenquimatoso ou subcapsular, e hemoperitôneo, pela laceração da cápsula de Glisson. Tipicamente, manifestam-se dentro de 24 horas após o procedimento.

Complicações menores podem ocorrer, como dor localizada e hipotensão transitória por reação vagal. Há poucos relatos na literatura de hematomas hepáticos resultando em complicações graves ou morte após biópsia hepática. ${ }^{8,11,12}$ Ahmed et al. relataram um caso de óbito resultante de hemorragia por pseudoaneurisma de artéria hepática, cuja necrópsia revelou necrose de praticamente todo lobo hepático direito. ${ }^{11}$

Nissen et al. relataram um caso de insuficiência hepática aguda após biópsia percutânea guiada por US em fígado transplantado, causada por hematoma expansivo com cápsula hepática intacta. Nesse caso, houve necessidade de novo transplante ortotópico total de fígado pela insuficiência hepática fulminante. Durante o retransplante, foi observado grande hematoma intracapsular com necrose de $90 \%$ do parênquima hepático e trombose de artéria hepática. ${ }^{8}$

É necessária monitorização após a realização do procedimento para detectar precocemente possíveis complicações.

Em nosso serviço, é estabelecido repouso relativo de seis horas após o procedimento, sendo monitorizada a pressão arterial e frequência cardíaca do paciente a cada 60 minutos, sendo mantido jejum durante esse período. Após esse tempo, é realizada avaliação laboratorial para avaliar a hemoglobina sérica e o coagulograma, bem como a dosagem das enzimas hepáticas. Somente após, o paciente recebe alta hospitalar.

Pouco tem sido descrito na literatura sobre biópsias em fígados transplantados. Nesses pacientes a biópsia hepática percutânea continua sendo o padrão ouro para o diagnóstico de rejeição. A biópsia permite a avaliação anatomopatológica, interpretando assim testes de função hepática alterados, ajuste das doses de imunossupressores, além de diagnosticar doença atual ou recidiva viral. ${ }^{13}$

Utilizamos em nossa prática a agulha de corte do tipo Tru-cut $14 \mathrm{G}$, com punção realizada guiada por US. A literatura corrobora que há menor chance de complicações com uso da agulha do tipo Tru-cut em relação à agulha de aspiração, ${ }^{3,4}$ assim como com a uso de ultrassonografia. ${ }^{2,3}$ Além disso, todas as biópsias percutâneas são executadas por radiologistas ou gastroenterologistas especializados, tanto no procedimento quanto na ultrassonografia.

$\mathrm{Na}$ maioria dos casos de sangramento após o procedimento, o tratamento conservador é suficiente, com repouso relativo, transfusão de componentes sanguíneos e internação hospitalar para observação 
clínica e laboratorial. Caso haja manutenção de instabilidade hemodinâmica, pode haver necessidade de angiografia com embolização, ou ainda de cirurgia. ${ }^{3,8,13}$ No caso descrito neste relato, acreditamos que a boa evolução clínica observada ocorreu devido a todos os fatores pontuados acima, com boa avaliação laboratorial prévia ao procedimento, protocolo bem estabelecido de observação clínica posterior à biópsia, tendo sido esta realizada por profissionais habilitados, e com pronta identificação do diagnóstico da complicação, além de seu imediato tratamento, nesse caso, conservador e altamente efetivo.

A biópsia transjugular é uma alternativa em pacientes com alto risco de sangramento, descrita em 1964. É realizada após punção venosa transjugular para acessar as veias hepáticas. Suas indicações compreendem coagulopatia grave, trombocitopenia, ascite de grande monta, obesidade mórbida, necessidade de outros procedimentos invasivos hemodinâmicos ou falha da biópsia percutânea. ${ }^{1,13,14}$ Porém, a habilidade operacional por profissionais certificados em técnicas endovasculares é essencial, mas menos disponíveis em nosso meio. Além disso, por vezes, as amostras adquiridas são insuficientes para diagnóstico anatomopatológico em comparação com as adquiridas por biópsia percutânea. Esse método também é sujeito a complicações, sendo a principal o sangramento, mas também relacionadas a punção venosa jugular e a passagem transatrial do cateter.1,13,14 Portanto, ainda não é método padrão e a biópsia percutânea é creditada como melhor método.

\section{CONCLUSÃO}

As complicações pós-biópsia após transplante de fígado, como o hematoma volumoso que aconteceu pós-biópsia hepática, pode ser conduzido de forma conservadora.

\section{ABSTRACT}

Percutaneous liver biopsy is a routinely used procedure in the postoperative period of the liver transplantation to investigate increased liver enzymes with a complication rate between $0.9 \%$ and $3.7 \%$, being the hemorrhage the most common. It is reported here the case of a 52-year-old man diagnosed with cirrhosis caused by hepatitis $C$ and alcohol who underwent liver transplantation under the Piggy Back technique 20 months ago. He had an uneventful postoperative period and started the follow-up at the Unicamp Hospital of Clinics (HC-Unicamp). Due to an unexplained elevation in the liver enzymes despite the adjustment of the immune suppression, the choice was submitting him to an inpatient percutaneous liver biopsy in order to assess a possible cellular rejection. One day after the biopsy, he presented severe abdominal pain and a significant increase in the aminotransferases associated with a 3 point decrease in the hemoglobin. A computerized abdomen scan tomography showed a major hepatic subcapsular hematoma. There was good outcome with non-operative treatment of the hematoma which required only one unit transfusion of packed red blood cells without needing other hemotherapic transfusion. He stayed at the hospital for observation for seven days, and he was discharged in good condition, restarting the outpatient-based follow up. Percutaneous liver biopsy is an invasive but very important procedure in the assessment and follow-up of the liver transplanted patients which may influence the immunosuppressive therapy as well as to diagnose the HCV recurrence and enable to treat such cases. Nevertheless, it is not free from complications inherent to the procedure, as reported here, and its indication should always be thoroughly evaluated, as it can be observed with no surgical procedure.

Keywords: Biopsy; Fine-Needle; Liver Transplantation; Hematoma; Complications. 


\section{REFERÊNCIAS}

1. Behrens G, Ferral H. Transjugular liver biopsy. Semin Intervent Radiol. 2012;29:111-7.

2. Mueller M, Kratzer W, Oeztuerk S, Wihelm M, Mason RA, Mao R, et al. Percutaneous ultrasonographically guided liver punctures: an analysis of 1961 patients over a period of ten years. BMC Gastroenterol. 2012;12:173.

3. Van Thiel DH, Gavaler JS, Wright H, Tzakis A. Liver biopsy. Its safety and complications as seen at a liver transplant center. Transplantation. 1993;55(5):1087-90.

4. Boyer JL. Outpatient liver biopsy: how safe is it? Ann Internal Med. 1993;118(2)150-3.

5. Weigand K, Weigand K. Percutaneous liver biopsy: retrospective study over 15 years comparing 287 inpatients with 428 outpacients. Gastroenterol Hepatol. 2009;24:792-9.

6. Von Frerichs FT. Berlin; Über den diabetes. Hirschwald, 1884.

7. Menghini G. One-second needle biopsy of the liver. Gastroenterol. 1958;35:190-9.

8. Nissen NN, Geller SA, Klein A, Colquhoun S, Yamini D, Tran TT, et al. Percutaneous liver biopsy after living donor liver transplantation resulting in fulminant hepatic failure: the first reported case of hepatic compartment syndrome. Transplantation. Doi: 10.1155/2010/273578.
9. Bubak ME, Porayko MK, Krom RAF, Wiesner RH. Complications of liver biopsy in liver transplant patients: increased sepsis associated with choledochojejunostomy. Hepatol. 1991;14(6):1063-5.

10. Larson AM, Chan C, Wartelle CF. Infection complicating percutaneous liver biopsy in liver transplant recipients. Hepatol.1997;26(6):1406-9.

11. Whittle Jr TS. Fatal intrahepatic hemorrhage after percutaneous liver biopsy using a Menghini needle: a rare but real complication. South Med J. 1977;70(11);1355-6.

12. Ahmed A, Samuels EB, Cheung, RC. . Delayed fatal hemorrhage from pseudoaneurysm of the hepatic artery after percutaneous liver biopsy. Am J Gastroenterol. 2001;96(1):233-7.

13. Van Ha TG. Liver biopsy in liver transplant recipients. Transplant Intervent. 2004;21(4):271-4.

14. Dohan A, Guerrache Y, Boudiaf M, Gavini JP, Kaci $R$, Soyer P. Transjugular liver biopsy: indications, technique and results. Diagn Irtervent Imaging. 2014 Jan;95(1):11-5 